\title{
Metabolic Management during Critical Illness: Glycemic Control in the ICU
}

\author{
Shyoko Honiden, MSc, MD ${ }^{1}$ Silvio E. Inzucchi, MD² \\ ${ }^{1}$ Department of Medicine, Section of Pulmonary and Critical Care \\ Medicine, Yale University School of Medicine, New Haven, \\ Connecticut \\ 2 Department of Medicine, Section of Endocrinology and Metabolism, \\ Yale University School of Medicine, New Haven, Connecticut
}

\begin{abstract}
Address for correspondence Shyoko Honiden, MSc, MD, 333 Cedar Street, PO Box 208057, New Haven, CT 06520-8057

(e-mail: shyoko.honiden@yale.edu).
\end{abstract}

Semin Respir Crit Care Med 2015;36:859-869.

\begin{abstract}
Keywords

- hyperglycemia

- hypoglycemia

- critical illness

- glycemic control

- insulin resistance

Hyperglycemia is a commonly encountered metabolic derangement in the ICU. Important cellular pathways, such as those related to oxidant stress, immunity, and cellular homeostasis, can become deranged with prolonged and uncontrolled hyperglycemia. There is additionally a complex interplay between nutritional status, ambient glucose concentrations, and protein catabolism. While the nuances of glucose management in the ICU have been debated, results from landmark studies support the notion that for most critically ill patients moderate glycemic control is appropriate, as reflected by recent guidelines. Beyond the target population and optimal glucose range, additional factors such as hypoglycemia and glucose variability are important metrics to follow. In this regard, new technologies such as continuous glucose sensors may help alleviate the risks associated with such glucose fluctuations in the ICU. In this review, we will explore the impact of hyperglycemia upon critical cellular pathways and how nutrition provided in the ICU affects blood glucose. Additionally, important clinical trials to date will be summarized. A practical and comprehensive approach to glucose management in the ICU will be outlined, touching upon important issues such as glucose variability, target population, and hypoglycemia.
\end{abstract}

Hyperglycemia is a commonly encountered metabolic disturbance among a heterogeneous group of patients with critical illness. In a study conducted between 2012 and 2013 in an Australian medical-surgical intensive care unit (ICU), among 1,000 patients examined, the majority of patients $(\sim$ $80 \%$ ) became hyperglycemic at some point during the first 48 hours of admission (defined as fasting blood glucose [BG] $\geq 126 \mathrm{mg} / \mathrm{dL}$, or random BG $\geq 200 \mathrm{mg} / \mathrm{dL}$ ). ${ }^{1}$ Prevalence of critical illness-associated hyperglycemia has been reported as being even higher in other studies. ${ }^{2}$ Hyperglycemia is an evolutionarily highly preserved response during periods of stress observed across many species and, to a degree, the old adage that it is "a compensatory mechanism to provide fuel to vital organs," may be in part true. While many studies have highlighted the epidemiological association between hyperglycemia and increased morbidity and mortality in numerous disease states, ${ }^{3-5}$ there have been, to date, somewhat discrepant results from randomized trials that have assessed whether controlling glucose intensively improves clinical outcomes. ${ }^{6-10}$ Added to this has been the complexity of factoring in related issues pertaining to optimal BG targets, any superimposed risks of hypoglycemia, glucose variability, questions about appropriate target populations (which might include the presence or absence of preexisting diabetes mellitus), and the method by which glucose control is attained (i.e., which insulin infusion protocol is implemented). Recent studies have also highlighted the relevance of preexisting glycemic milieu for each patient, as a higher "metabolic penalty" may be incurred by an individual with true de novo stress hyperglycemia. ${ }^{111-14}$ The seemingly
Issue Theme Controversies and Evolving Concepts in Critical Care; Guest Editors: Wassim H. Fares, MD, MSc, and Mark D. Siegel, MD
Copyright $\odot 2015$ by Thieme Medical Publishers, Inc., 333 Seventh Avenue, New York, NY 10001, USA. Tel: +1(212) 584-4662. 
simple question of what BG threshold should be used-above which stress hyperglycemia could be considered maladaptive-has therefore been a source of much debate and controversy in the past decade, as we have come to better appreciate the nuances of metabolic management of critically ill patients. The following brief review will highlight the physiologic consequence of uncontrolled glycemic excursions, the BG target to consider, and how to best achieve glycemic control in the ICU. Furthermore, the occurrence of hyper- as well as hypoglycemia is indirectly linked to nutritional intake during critical illness. Related concepts pertaining to ICU nutrition in the context of metabolic management will also be reviewed.

\section{The Biology of Hyperglycemia during Critical Illness}

\section{Why Hyperglycemia Develops and How Hyperglycemia Affects Critical Cellular Pathways}

While a comprehensive review of glucose and insulin dynamics during critical illness is beyond the scope of this review, there is a complex interplay between patient factors, cellular derangements, nutrition, and glycemia in the critically ill. Hyperglycemia during illness is fueled by a state of insulin resistance. Counter-regulatory hormones (such as catecholamines, cortisol, glucagon) and elevated levels of cytokines (such as interleukin-1 [IL-1], IL-6, tumor necrosis factor- $\alpha$ [TNF- $\alpha]$ ) promote hyperglycemia mainly through the induction of insulin resistance, which results in impaired peripheral glucose uptake and increased endogenous glucose production (mainly through hepatic gluconeogenesis and glycogenolysis). ${ }^{15-17}$ In the fasting state, glycogen stores are eventually depleted, but gluconeogenesis remains unimpaired.

Hyperglycemia, in turn, has several immunomodulatory effects that are potentially deleterious in critical illness, such as increased production of anti-inflammatory cytokines like IL-10, and impairment of polymorphonuclear neutrophil function, resulting in decreased intracellular bactericidal activity, opsonic activity, and innate immunity. ${ }^{18-21}$ Hyperglycemia can also promote inflammation by increasing proinflammatory cytokines such as TNF- $\alpha$, IL$1 \beta,-6,-8$, and $-18,{ }^{22}$ increasing leukocyte adhesion molecules, inducing $\mathrm{NF}-\mathrm{KB},{ }^{23}$ and promoting a procoagulant state. ${ }^{18-21}$ Hyperglycemia additionally exacerbates oxidative stress. $^{24-26}$

\section{The Link between Oxidant Stress, Organ Dysfunction, Mitochondrial Health, and Glycemia}

Increasingly recognized is the coupling between oxidant stress and mitochondrial DNA injury. Mitochondrial damage results in an inability to maintain basic cellular functions and has been associated with the onset of multiorgan dysfunction syndrome. Effective removal of damaged mitochondria through mitophagy and regeneration of healthy mitochondria is associated with better outcomes. ${ }^{27}$ Interestingly, some studies suggest that this core cellular homeostatic function is worsened in the presence of hyperglycemia. ${ }^{28}$

\section{The Role of Immobility and Insulin Resistance}

Exercise-stimulated glucose uptake in skeletal muscle plummets as patients become bedbound. ${ }^{29}$ As a corollary, a recent study tested whether an early ICU mobilization program can increase glucose transport in contracting skeletal muscles and thereby ameliorate hyperglycemia. This concept was supported by the observation that exercise has an ability to reduce inflammation and improve insulin resistance. ${ }^{30}$ For example, among healthy patients with type 2 diabetes mellitus, a single bout of 45 to 60 minutes of exercise enhanced glucose transport and insulin sensitivity by up to 20 hours after exercise. ${ }^{31}$ In a secondary analysis of data derived from a randomized trial studying early mobility, patients with and without ICU weakness achieved identical glycemic control (median BG $131 \mathrm{mg} / \mathrm{dL}$ in both groups, with glucose managed per standardized insulin protocol), but those randomized to early ICU mobilization (average therapy session of $25 \mathrm{mi}-$ nutes) had reduced insulin resistance as evidenced by lower daily insulin requirements. ${ }^{32}$ Additional studies are needed to validate this concept.

\section{The Relationship between Enteral Feeding and Hyperglycemia}

Normally after eating a meal, serum glucose increases and insulin is released. Together, these events trigger downregulation of glucagon release. During critical illness, this feedback loop becomes impaired. Meal ingestion normally also triggers a complex entero-hormonal response involving glucose-like peptide-1 (GLP-1), gastric inhibitory peptide (GIP), cholecystokinin (CCK), ghrelin and peptide YY. These hormones regulate intestinal motility, nutrient absorption, as well as gallbladder and pancreatic islet cell function. GIP and GLP-1 are incretin hormones secreted by the gut; they potentiate $\beta$ cell insulin secretion in a glucose-dependent fashion. GLP-1 also suppresses glucagon release, which, in turn, results in a reduction in hepatic glucose production. After a bolus meal, these hormones experience a brisk rise followed by a return to basal levels, but this pulsatile hormonal response to nutrition is dampened with continuous tube feeding (when compared with bolus, intermittent feeding) and also associated with greater insulin resistance in an experimental model in healthy piglets. ${ }^{33,34}$ In reality, no mammalian species eats throughout a 24-hour period, and there may be an underappreciated metabolic implication to this standard practice in the ICU. ${ }^{35}$ Continuous enteral feeding may also inadvertently predispose patients to hypoglycemia due to interruptions (which are sometimes prolonged and frequent) related to procedures and tests in the ICU. Further research is needed to define the best approach to providing sufficient nutrition during critical illness.

While gastric emptying delay is recognized as a consequence of long-standing diabetes and primarily thought of as a chronic neuropathic change in the gut, there is now ample evidence that acute hyperglycemia can also slow down gastric emptying in both healthy subjects and in those with diabetes. ${ }^{36-38}$ Critically ill patients, already prone to ileus, may therefore be additionally disadvantaged when confronted by poorly controlled glucose concentrations. In one study, 95 
consecutive feed-intolerant critically ill patients with no known history of diabetes were compared with 50 feedtolerant critically ill patients in a medical-surgical ICU. ${ }^{39}$ The groups were matched for age, gender, body mass index, APACHE score, as well as other important variables such as receipt of opioid, inotropic, and sedative medications. Feedintolerant patients were more likely to have higher peak BG before and during feeding, demonstrated more glucose variability, and had more episodes of BG $>180 \mathrm{mg} / \mathrm{dL}$ which tended to be of longer duration compared with feed-tolerant patients, despite receiving similar amounts of insulin. Prokinetic agents, such as intravenous erythromycin, are sometimes used for refractory feed intolerance in the ICU. Interestingly, some studies have suggested degree of glycemia to impact treatment responses and have demonstrated significantly muted response to prokinetic agents during hyperglycemia (vs. euglycemia) in experimental models. ${ }^{40}$ As ileus worsens and feeding attempts get increasingly interrupted, this also likely places the patient at a higher risk for developing hypoglycemia and greater glucose variability (both concepts discussed separately later), as attempts are made to control glucose excursions without a steady provision of nutrition.

\section{Summative Findings from Randomized Studies of Glycemic Control for ICU Patients}

The first randomized control study examining the effects of stringent glucose management in the ICU was published in 2001. It demonstrated that intensive insulin therapy, with a BG goal between 80 and $110 \mathrm{mg} / \mathrm{dL}$, improved meaningful outcomes among ventilated surgical ICU patients. The absolute reduction in ICU mortality was 3.4\% (relative risk [RR], $-42 \%)$ and the benefit was amplified (9.6\% absolute reduction; RR $-48 \%$; number needed to treat $\sim 10$ ) among those requiring ICU level care for more than 5 days. ${ }^{10}$ The follow-up medical ICU study by the same group, however, showed no mortality benefit in the intention to treat analysis, although a significant mortality improvement was again demonstrated in the prespecified target population of patients who required an ICU stay of 3 days or more. ${ }^{9}$ From a practical perspective, however, this subset was difficult to pre-identify at the time of ICU admission. A subsequent pooled analysis of the medical ICU and the original surgical ICU cohorts from these investigations showed improvements in mortality without any observable harm. ${ }^{41}$

In comparison, the multicenter European trial Glucontrol and the German sepsis trial VISEP reported frequent protocol violations and high hypoglycemia rates, and as a result were terminated early. ${ }^{6,8,42}$ There were no differences in mortality observed between the conventional and the intensively treated groups in either of these studies. Finally, the most recently published NICE-SUGAR trial, the largest randomized controlled trial to date with more than 6,100 participants, seemed to favor "good but not tight" control with a BG target of 140 to $180 \mathrm{mg} / \mathrm{dL}^{7}$ In the end, the results seen in the original Belgian study have not been replicated, and in NICESUGAR, extremely tight control of BG (80-108 mg/dL) was associated with slightly higher 90-day mortality when compared with those patients with moderate control (144-180 $\mathrm{mg} / \mathrm{dL}$ ) (27.5 vs. 24.9\%; odds ratio [OR] for mortality with intensive control 1.14; 95\% confidence interval [CI]: 1.02$1.28 ; p=0.02$ ), although reasons for this observation were not clear. Summary statistics from the large multicentered studies can be found in - Table 1. Follow-up evaluations from NICE-SUGAR found that severe hypoglycemia in both the intensive and standard care groups was associated with greater mortality. Finally, while there is significant heterogeneity between studies, the latest meta-analysis (which include results from NICE-SUGAR) concluded that very tight glucose control did not improve mortality for all-comer ICU patients. ${ }^{43}$ The pooled RR for death across the 26 studies included was 0.92 (95\% CI: 0.83-1.04), but when analysis was limited to surgical ICU patients, the RR was 0.63 (95\% CI: 0.440.91, however, not adjusting for multiple testing), suggesting there still may be some benefit in selected populations (-Fig. 1). To reflect the cumulative evidence to date, the latest recommendations from both the American Association of Clinical Endocrinologists and the American Diabetes Association suggest a target BG range of 140 to $180 \mathrm{mg} / \mathrm{dL}$ for most ICU patients, with greater benefit derived with an average glucose at the lower end of this range. ${ }^{44}$ The third edition of the Surviving Sepsis Campaign International guidelines from 2012 largely mirror these recommendations, suggesting treatment initiation when two or more consecutive BG measurements exceed $180 \mathrm{mg} / \mathrm{dL}$. While setting an upper target limit of $180 \mathrm{mg} / \mathrm{dL}$, the most recent Surviving Sepsis guidelines did not strictly predefine a target range or lower glucose threshold level given the lack of clear evidence to support one target range over another, and simply added that avoidance of hypoglycemia was paramount. ${ }^{45}$

\section{The Practical and Comprehensive Approach to Glycemic Management in the ICU}

\section{The Target Population}

As reflected in the latest meta-analysis and expert recommendations, despite somewhat disparate results of studies, reverting back to an era of lax glucose control is unacceptable, and for most ICU patients moderate glucose control (140-180 $\mathrm{mg} / \mathrm{dL}$ ) is appropriate. One may argue, however, that, in reality, the "one-size-fits-all" approach is too imprecise, as the physiology and needs during critical illness are quite heterogeneous.

One factor to consider pertains to the presence or absence of preexisting diabetes mellitus. Indeed, not all hyperglycemia is equally associated with adverse outcomes during acute illness. $^{12,14,46,47}$ Patients with no prior diagnosis of diabetes have worse outcomes for the same degree of hyperglycemia compared with patients with known diabetes mellitus. The mechanism to explain this phenomenon is still unknown, but confounding factors as well as the impact of the preexisting metabolic milieu are likely relevant. To support this latter possibility, at a cellular level acute fluctuations in BG levels (particularly when truly transient and unrelated to preexisting diabetes) appear to induce more ischemic injury, 


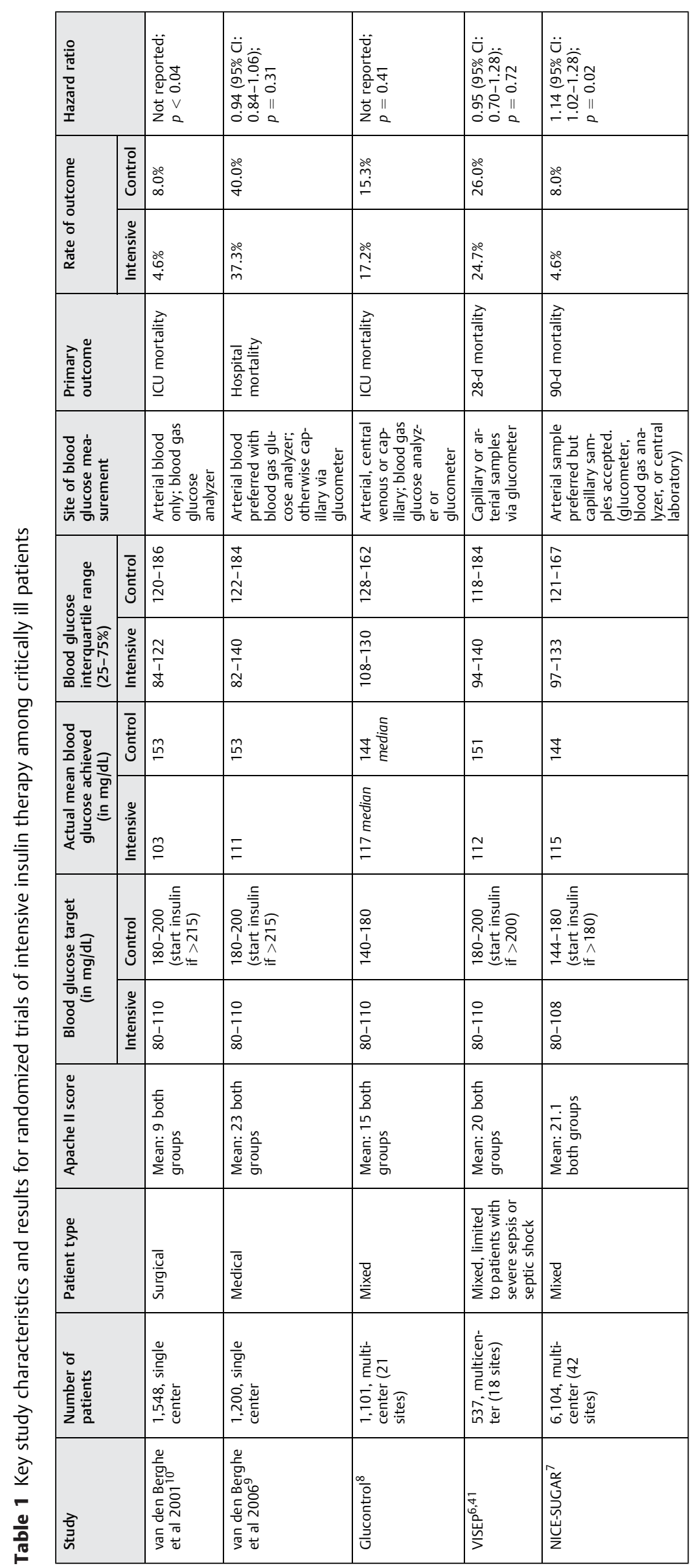




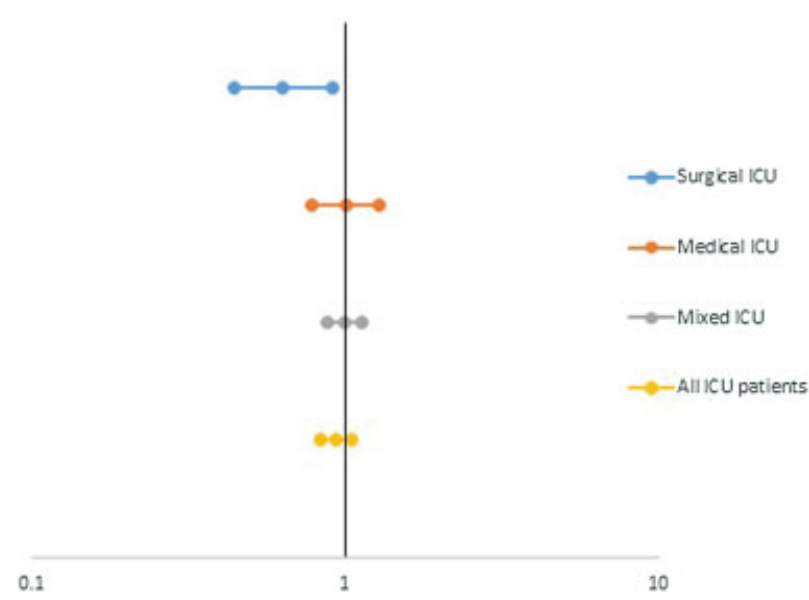

Fig. 1 Results from the post-NICE SUGAR meta-analysis: risk of 90-day mortality in clinical studies comparing conventional glycemic control and intensive insulin therapy, stratified by ICU type. (Data adapted from Griesdale et al. ${ }^{43}$ )

inflammation, endothelial dysfunction, cellular apoptosis, and oxidative stress. ${ }^{48,49}$ Conversely, those with chronic hyperglycemia may have compensatory mechanisms in place that provide protection from acute hyperglycemia-related cellular damage. In support, recent ICU studies have shown that among hyperglycemic patients, those with higher preadmission hemoglobin A1c (HbA1c) have significantly lower mortality compared with patients with lower HbA1c. ${ }^{11,50}$ Some have suggested that the greatest benefit from intensive insulin treatment may be reaped by those with newly discovered hyperglycemia or those with a low premorbid HbA1c levels, ${ }^{9,41,51}$ but such recommendations do not stem from properly designed clinical trials and are therefore not part of prevailing guidelines at this time.

Another example of a subset that may require special attention are neurologically injured patients. Modest hyperglycemia (in the range of $150-170 \mathrm{mg} / \mathrm{dL}$ ) has been associated with morbidity and mortality in cerebral hemorrhage, ischemia, and trauma, ${ }^{52-55}$ and experimental animal models have shown that hyperglycemia can extend neuronal injury from ischemia. ${ }^{56}$ Despite these observations and success seen in a limited number of neurosurgical patients in the original Leuven study, ${ }^{57}$ other studies that have examined intensive insulin therapy among patients with cerebral injury have not shown consistent improvements in survival or functional outcome (but perhaps a modest improvement in infection rates). ${ }^{58-60}$ A possible reason may be related to exquisite sensitivity to even moderate hypoglycemia during acute brain injury. The balancing act between high and low BG and finding the possibly very narrow optimal range is particularly challenging in this cohort. In a small, observational study focusing on patients with acute brain injury, one group suggested that brain glucose was reduced by up to $70 \%$ among patients intensively treated with insulin (compared with 15\% in the standard control group) using data obtained from cerebral microdialysis and PET imaging. ${ }^{61}$ The brain hypoglycemic threshold corresponded in this study to a BG level less than $80 \mathrm{mg} / \mathrm{dL}$ which is typically considered "safe."

\section{Monitoring Performance: Beyond Measuring the Average Glucose}

\section{The Role of Hypoglycemia}

Tighter glucose control, no matter how well executed the protocol, will almost always lead to higher rates of hypoglycemia and some patients, such as the aforementioned braininjured patients, may be particularly sensitive to the harmful effects of low BG concentrations. Published severe hypoglycemia rates (typically defined as the percent of patients experiencing at least one $\mathrm{BG}<40 \mathrm{mg} / \mathrm{dL}$ ) differ widely, and may be as low as $5.1 \%$ among surgical ICU patients in the original Leuven study ${ }^{10}$ to as high as nearly one in five in the medical $\mathrm{ICU}^{9}$ (-Table 2). There is debate about the clinical significance of these hypoglycemic events, particularly when identified quickly and appropriately addressed in a closely monitored ICU setting. Some believe that iatrogenic hypoglycemia is yet another marker of poor outcome and severity of illness and that it does not directly cause morbidity and mortality. In a large retrospective cohort study involving nearly 17,000 patients hospitalized for acute myocardial infarction, the likelihood of death in the hospital rose significantly when mean glucose levels fell below $70 \mathrm{mg} / \mathrm{dL}$ compared with those who had mean levels between 100 and 109 $\mathrm{mg} / \mathrm{dL}$ (OR: $6.4 ; p=0.01$ ). ${ }^{62}$ In this study, it was spontaneous hypoglycemia, rather than insulin-induced hypoglycemia, that was associated with higher mortality. These data suggest that iatrogenic hypoglycemia may not carry with it significant risk. Instead, the predisposition to developing hypoglycemia in the absence of glucose-lowering therapy (as may occur in sepsis, hepatic failure, renal failure, and adrenal insufficiency) may simply identify a more vulnerable patient population.

Meanwhile, in a case-control study of a mixed medicalsurgical ICU, hypoglycemia was associated with an increased risk of death after multivariate analysis (OR: 2.28 [CI: 1.413.7]) but on balance, the benefit reaped from glycemic control appeared to outweigh this risk. ${ }^{63}$ Others, such as Vriesendorp and colleagues, have shown no association between hypoglycemia and mortality. ${ }^{64}$

Several commonly encountered factors have been associated with an increased risk of hypoglycemia, which may help identify patients who require closer monitoring: bicarbonate-based fluid during continuous venovenous

Table 2 Hypoglycemia rates for key randomized trials of intensive insulin therapy among critically ill patients

\begin{tabular}{|c|c|c|}
\hline \multirow[t]{2}{*}{ Study } & \multicolumn{2}{|c|}{$\begin{array}{c}\text { Hypoglycemia rate }(\leq 40 \\
\mathrm{mg} / \mathrm{dL})\end{array}$} \\
\hline & Intensive(\%) & Control (\%) \\
\hline van den Berghe et al $2001^{10}$ & 5 & 0.7 \\
\hline van den Berghe et al $2006^{9}$ & 18.7 & 3.1 \\
\hline Glucontrol $^{8}$ & 8.7 & 2.7 \\
\hline VISEP 6,41 & 17.0 & 4.1 \\
\hline NICE-SUGAR ${ }^{7}$ & 6.8 & 0.5 \\
\hline
\end{tabular}


hemofiltration (CVVH), decreased rate or interruption of nutritional support, need for inotropic support, sepsis, female sex, prior diabetes, and octreotide use. ${ }^{65}$ Septic patients may be at risk for delayed recognition of hypoglycemia when capillary finger stick measurements are used to titrate insulin during low perfusion states. ${ }^{66-68}$

Despite lack of definitive data proving the dangers of hypoglycemia encountered in the ICU, it is important to remember that prolonged severe hypoglycemia can undoubtedly deplete astrocyte glycogen stores and lead to neuronal cell death and permanent brain injury. Concurrent administration of sedatives and the debilitated critically ill state may mask the usual symptoms of low BG such as anxiety, diaphoresis, tachycardia, palpitations, and perceptible change in cognitive function and may lead to delayed recognition if BG checks are not done at frequent intervals. Furthermore, one isolated hypoglycemic event may be well tolerated, but frequent and repeated episodes may deplete glycogen stores over time and place a patient at risk for neuronal injury even if each episode is of relatively short duration. The long-term neurocognitive consequences of recurrent or severe hypoglycemia are not well understood.

\section{The Role of Glucose Variability}

How we control glucose matters, and glucose variability may be as important as the mean glucoses achieved. Egi and colleagues demonstrated that glucose variability (defined as the standard deviation of glucose) was an important independent predictor of mortality-more powerful than mean glucose concentrations among a heterogeneous group of ICU patients. ${ }^{69}$ In another study, there was a statistically significant difference in magnitude of change between two successive BG values when comparing survivors to nonsurvivors. ${ }^{70}$ Notably, there was no difference in the conventionally reported metric of glucose control in terms of mean BG between the two groups. In a study reported by Krinsley, among patients with a mean BG level between 70 and $99 \mathrm{mg} /$ $\mathrm{dL}$ during their ICU stay, mortality was $5.9 \%$ if this was achieved with the least amount of glucose variability ${ }^{71}$; for the same degree of "excellent" control, mortality rose to $30.1 \%$ among patients with the highest quartile of glucose variability. For patients with a mean BG ranging between 80 and 110 $\mathrm{mg} / \mathrm{dL}$, the target range utilized in the Leuven study and in subsequent trials, mortality ranged from 4.2 to $27.5 \%$ depending on the degree of glucose variability. While the incidence of severe glucose variability (defined as a BG measurement below $81 \mathrm{mg} / \mathrm{dL}$ and above $216 \mathrm{mg} / \mathrm{dL}$ occurring within 24 hours of ICU admission) appeared to be rare (occurring in only $\sim 3 \%$ of patients) in a large retrospective cohort study examining more than 66,000 adult ICU admissions, the presence of significant BG variability was associated with higher covariate-adjusted ICU $(1.5,95 \% \mathrm{CI}: 1.4-1.6)$ and hospital mortality (1.4, 95\% CI: 1.3-1.5) when compared with the presence of hypoglycemia (BG below $81 \mathrm{mg} / \mathrm{dL}$ ) only or when compared to patients without either factor (i.e., hypoglycemia and significant variability). ${ }^{72}$

When patients develop hypoglycemia on intensive insulin therapy, most protocols call for administration of intravenous dextrose. In the Leuven protocol, $10 \mathrm{~g}$ of dextrose was given when the BG dropped below $40 \mathrm{mg} / \mathrm{dL}$. With BG rebounding shortly after administration of dextrose, such a protocol may therefore contribute to glucose variability. Therefore, when hypoglycemia rates are high, as was seen in the Leuven MICU study at 18.7 and at $17 \%$ in the VISEP trial, it is possible that the consequent increases in glucose variability may be negating the potential beneficial effects of glucose control.

Biologically, in vitro experiments have shown that fluctuating glucose levels induce apoptosis more robustly than sustained hyperglycemia, cause endothelial activation, and lead to oxidative stress. ${ }^{48,49,73}$ Similarly in vivo, rapid fluctuation in BG is associated with oxidative stress, and this relationship may be more important than the level of chronic sustained hyperglycemia among type 2 diabetics. ${ }^{74}$ In type 1 diabetes, one interpretation of the results from the outpatient Diabetes Control and Complication Trial (DCCT) suggests that increased glucose variability is associated with higher rates of retinopathy in cohorts of patients at similar hemoglobin A1C strata. $^{75,76}$

Future studies need to examine and report the effect of glucose variability, as this may be an important metric to follow. With many such measures having been proposed by investigators, a standardized language and metric to describe "variability" should be established. Additionally, prospective evaluation is needed to clarify whether glucose variability is simply an epiphenomenon associated with severity of illness and the multiple interventions required in the sickest patients that lead to metabolic perturbations, including hypoglycemia, or whether it itself is a physiologic derangement in need of targeted intervention. We would add that a similar controversy has taken place for years regarding the chronic care of the diabetic patient. Yet, to date, there remains no compelling evidence that targeting glucose variability in these outpatients actually translates to improved clinical outcomes.

\section{Monitoring Glucose}

Any program of intensive glucose control necessitates accurate and precise BG concentration measurements. Currently, the "gold standard" in the inpatient setting is central laboratory measurement of plasma glucose, preferred over whole BG because it is less influenced by the prevailing hematocrit. The sample source may also affect the measured glucose value, with arterial blood, in the normal physiological range, typically registering $\sim 10 \mathrm{mg} / \mathrm{dL}$ higher than venous blood (and $\sim 5 \mathrm{mg} / \mathrm{dL}$ higher than capillary blood). Differences between these sites may be accentuated in the setting of significant hyperglycemia. Because of their overall convenience and rapid turnaround, capillary point-of-care (POC) ("finger stick") meters have become inculcated into the daily work flow of hospital wards and even intensive care units. These devices use the glucose oxidase or glucose dehydrogenase reactions to estimate the BG concentration. Increased catecholamines, uric acid, and bilirubin as well as the presence of certain drugs such as acetaminophen may interfere with glucose-oxidase reactions. ${ }^{77}$ Those that rely on the glucose dehydrogenase reaction are less subject to error 
due to interfering metabolites and medications, but they may detect sugars other than glucose, such as mannose, xylose, and icodextrin, which will lead to an overestimate of the reported BG concentration. ${ }^{77}$

Recently, the Food and Drug Administration (FDA) has challenged the appropriateness of using POC capillary meters in the hospital, as their accuracy has been validated mainly in the outpatient setting. The FDA currently endorses the standards of the International Organization for Standardization (ISO) in which $95 \%$ of glucose values measured by a device must fall within $15 \mathrm{mg} / \mathrm{dL}$ of the reference method (typically laboratory plasma glucose) for BG concentrations below 75 $\mathrm{mg} / \mathrm{dL}$ and within $20 \%$ for BG concentrations above $75 \mathrm{mg} /$ dL. ${ }^{77}$ In a proposed set of guidelines, the FDA would mandate more stringent requirements for hospital-use glucose meters, for example, $99 \%$ of all values falling within $7 \mathrm{mg} / \mathrm{dL}$ for BG below $70 \mathrm{mg} / \mathrm{dL}$ and within $10 \%$ for above $75 \mathrm{mg} / \mathrm{dL}^{78}$ In addition, meter manufacturers would need to provide validation data demonstrating accuracy and precision in a variety of hospitalized patient types, including the critically ill. ${ }^{79}$

Measuring capillary BG in the critically ill patient is indeed a challenge because commonly encountered physiologic disturbances can affect the measurements. For example, hypotension with resultant hypoperfusion and acidosis as well as severe anemia and hypothermia may introduce additional sources of error. ${ }^{80}$ In addition to accuracy concerns, finger stick monitoring is somewhat time intensive with regard to nursing work and uncomfortable for the patient, as lancing the fingertip is necessary for each measurement. Accordingly, there has been significant recent interest in adopting "continuous glucose monitoring (CGM)" systems for inpatient use, with the hope that these new devices might help achieve smoother glycemic control and avoid the metabolic consequences of hypoglycemia and glucose variability in critically ill patients. In recent years, these devices have gained popularity in the outpatient care for patients with type 1 diabetes, particularly those on insulin pumps or other intensive management strategies. There are, however, few data on their role in intensive care unit or other hospitalized patients.

At the time of this writing, in the United States, there are two FDA-approved outpatient interstitial CGM systems (Enlite [Medtronic, Minneapolis, MN] and G4 Platinum [DexCom, San Diego, CA]) and one approved for inpatient use but only in Europe (Sentrino [Medtronic]). These devices work through a subcutaneously inserted sensor that measures the interstitial glucose concentration, which reacts with glucose oxidase to generate hydrogen peroxide in a redox reaction similar to that of many capillary glucose meters. Reciprocally, hydrogen peroxide is oxidized, releasing electrons and creating a current-the amplitude of which corresponds to the glucose concentration. This information is then transmitted continuously through radio waves to a small display device (about the size of a pager), which can present it graphically, updated every 5 minutes. Each sensor has a life of 3 to 7 days, depending on the model. Periodic calibration via capillary BG is necessary, and current devices, which remain imperfect particularly in the hypoglycemic range, require that any decisions on insulin dose changes should be made only after the CGM glucose value (either high or low) is confirmed by a finger stick. In addition to the some of the same interferences faced by POC meters using glucose oxidase, measurement of interstitial glucose carries with it certain peculiarities. In steady state, interstitial glucose actually correlates well with ambient plasma glucose concentrations. However, it is very much influenced by the rate of change of plasma concentrations, capillary permeability, volume status, and proximate edema. Of specific importance is a lag phenomenon between the current plasma glucose reading and its interstitial counterpart amounting to approximately 15 to 30 minutes. The differences are most prominent when the BG is not in steady state, such as in the postprandial setting or after correction doses of insulin have been administered. ${ }^{81}$ Current CGM devices also provide warning alerts to the wearer regarding impending hypoglycemia and severe hyperglycemia. When worn consistently, they have been demonstrated to improve overall control and decrease hypoglycemic episodes in type 1 diabetes, in the outpatient setting. ${ }^{82}$

Of note, several intravascular devices are also under investigation. These indwelling units read ambient glucose in venous blood real time. ${ }^{83-86}$ Other devices categorized as "intravascular" actually draw blood to and through an external sensor, subsequently either recirculated or discarded. The GlucoScout (International Biomedical Ltd., Austin, TX) is now FDA-approved for sampling arterial or venous blood every 5 minutes. Of similar design is the GlucoClear sensor (Edwards LifeSciences, Irvine, CA), now approved in Europe. Of course, any truly indwelling device carries with it the risk of infection and thrombosis, as well as possible interference from other solutions being delivered through the same vascular access channel.

Several studies have assessed the role of CGM in the hospital setting, most in the ICU. ${ }^{87-93}$ The majority concluded that the new technology has accuracy comparable to when used in the outpatient setting, despite circumstances of tenuous hemodynamic status or the ongoing use of pressors. For example, retrospective analysis of CGM data from 174 ICU patients being tracked via arterial BG as the reference found a practical concordance of $\sim 99 \%$ when assessed by Clarke Error Grid analysis. ${ }^{94}$ That is, the paired results would have each resulted in the same clinical decisions in all but $1 \%$ of results. ${ }^{90}$ Others, however, have uncovered significant and concerning discordance specifically in the hypoglycemic range with high false-alarm rates. Rabiee and colleagues, for example, found that the CGM failed to detect up to $50 \%$ of hypoglycemic episodes in hospitalized patients as determined by finger stick BG. ${ }^{95}$ Also, a study looking at the performance of the Sentrino, the first subcutaneous CGM device designed specifically for hospital use, while finding good overall correlation to standard BG measures, called into question the reliability of its alarms, especially in the context of hypoglycemia. ${ }^{92}$ The system failed to identify 6 out of 24 (25\%) hypoglycemic events $<80 \mathrm{mg} / \mathrm{dL}$ as confirmed by central laboratory plasma venous values. Additionally, of 47 hypoglycemia alarms, 33 proved erroneous, resulting in a $70 \%$ false-alarm rate. As for the intravascular devices, preliminary 
investigations have demonstrated them to be reasonably accurate in the ICU setting with low rates of device-related complications, although mainly in small, short-term studies. ${ }^{83-86}$

Three randomized trials of CGM in the inpatient setting have been conducted to date. In two studies, by Boom et al $^{96}$ and Kopecký et al, ${ }^{91}$ no differences in glycemic control could be demonstrated with the more expensive CGM versus more conventional intermittent assessments either using arterial or capillary BG. Boom's data suggested the nursing time and costs may have been reduced, however, with CGM. In these two investigations, the standard insulin therapy protocols were being used with conventional BG monitoring. In the only such study that had adapted its insulin infusion protocol to match the more frequent and voluminous CGM data, Holzinger et al also found roughly equivalent overall glycemic control between the two groups. However, the rate of severe hypoglycemia was $1.6 \%$ in the CGM as compared with $11.5 \%$ in the control group, representing a RR reduction for these events of $86 \%{ }^{97}$

In summary, there are no solid data yet to support the use of CGM in their current form in the ICU. However, as these devices become more accurate, especially in the hypoglycemia range, it is conceivable that they will emerge as an important method by which critical care physicians can track their patients' glucose concentrations more closely than with intermittent laboratory or POC assessments. Of course, extensive comparative effectiveness and cost-effectiveness research will need to be conducted. Additionally, more advanced glucose control algorithms will need to be developed to take advantage of the significantly more data points provided by CGM so as to more precisely titrate insulin infusions. The goal would be better and safer glucose control, with less variability. In the future, it is also possible that highly accurate CGM sensors will be able to communicate directly with insulin infusion pumps, for a truly automated system (or "artificial" or "bionic pancreas"). ${ }^{98}$ These so-called closed loop devices are being actively investigated in outpatients with type 1 diabetes. They hold significant potential to radically change current treatment paradigms across the continuum of care in patients requiring insulin therapy for their diabetes.

\section{Considering the Influence of Nutrition on Glycemic Control}

In general, energy intake should be adjusted to avoid excessive glucose intake and overfeeding to minimize the emergence of hyperglycemia. Additionally, the hormonal milieu that fuels overexuberant gluconeogenesis can also predispose to protein catabolism. Literature suggests that this catabolic state is not necessarily reversed with full caloric intake. With this in mind, some studies have shown that provision of $50 \%$ of energy needs is sufficient to maintain the same nitrogen balance as "full" feeds, and yet able to lower the incidence of overt hyperglycemia. While the optimal caloric intake during critical illness is not known, at least early on during an ICU stay, some degree of temporary underfeeding may be safe and can help control glucose excursions especially in the setting of a severely insulin-resistant state. ${ }^{99,100}$ On a related note, managing glucose effectively may also ameliorate protein catabolism, as there is preliminary evidence to suggest that moderate glucose control promotes less negative nitrogen balance among medical ICU patients. ${ }^{101}$

While temporary mild underfeeding might be acceptable, avoidance of overt malnutrition is of obvious importance. At least among critically ill children, a prospective cohort study suggested that both hyper- and hypoglycemia were associated with worsened morbidity and mortality even after adjustment for disease severity in malnourished (but not well-fed) participants. ${ }^{102}$ A similar study in adults have not been performed, but it is conceivable that the metabolic impact of hyper- as well as hypoglycemia during critical illness may differ depending on the nutritional backdrop of the patient.

Certainly, provision of nutrition mitigates the risks associated with severe hypoglycemia, and a prolonged fasting state may in fact worsen insulin resistance. In one recent small study, a modest amount of enteral nutrition (e.g., $60 \%$ of goal rate) was demonstrated to be more effective at significantly reducing the incidence of hypoglycemic events (defined as $\mathrm{BG}<50 \mathrm{mg} / \mathrm{dL}$ ), compared with dextrose containing intravenous solutions. A relatively large volume $(\sim 150 \mathrm{~mL} /$ hour of $5 \%$ dextrose solution) was required to achieve similar hypoglycemia event rates. ${ }^{103}$

Provision of lower glycemic index carbohydrates, monounsaturated fatty acids, and fiber may help improve glycemic control and lower insulin requirements. ${ }^{104,105}$ Although earlier trials suggested that glutamine and antioxidant-enriched nutritional support may improve survival with the added benefit of improved glycemic control, ${ }^{106,107}$ this practice is no longer recommended based on the results from the recent, large multicenter randomized trial in which early glutamine administration was associated with a trend toward worsened mortality (OR: $1.28,95 \% \mathrm{CI}: 1.00-1.64, p=0.05){ }^{108}$

\section{Conclusion}

Hyperglycemia is commonly encountered in the ICU. We now have a better understanding about critical cellular pathways that can become deranged with prolonged and uncontrolled hyperglycemia, and we are just beginning to appreciate the complex relationship between nutritional status, protein catabolism, and insulin-resistant states. For most patients, moderate glycemic control is appropriate, and new technologies such as continuous glucose sensors may help alleviate the risks associated with excessive glucose variability as well as severe hypoglycemia.

\section{References}

1 Plummer MP, Bellomo R, Cousins CE, et al. Dysglycaemia in the critically ill and the interaction of chronic and acute glycaemia with mortality. Intensive Care Med 2014;40(7):973-980

2 Cely CM, Arora P, Quartin AA, Kett DH, Schein RM. Relationship of baseline glucose homeostasis to hyperglycemia during medical critical illness. Chest 2004;126(3):879-887 
3 Krinsley JS. Association between hyperglycemia and increased hospital mortality in a heterogeneous population of critically ill patients. Mayo Clin Proc 2003;78(12):1471-1478

4 Laird AM, Miller PR, Kilgo PD, Meredith JW, Chang MC. Relationship of early hyperglycemia to mortality in trauma patients. J Trauma 2004;56(5):1058-1062

5 Rovlias A, Kotsou S. The influence of hyperglycemia on neurological outcome in patients with severe head injury. Neurosurgery 2000;46(2):335-342, discussion 342-343

6 Brunkhorst FM, Engel C, Bloos F, et al; German Competence Network Sepsis (SepNet). Intensive insulin therapy and pentastarch resuscitation in severe sepsis. N Engl J Med 2008;358(2): 125-139

7 Finfer S, Chittock DR, Su SY, et al; NICE-SUGAR Study Investigators. Intensive versus conventional glucose control in critically ill patients. N Engl J Med 2009;360(13):1283-1297

8 Preiser JC, Devos P, Ruiz-Santana S, et al. A prospective randomised multi-centre controlled trial on tight glucose control by intensive insulin therapy in adult intensive care units: the Glucontrol study. Intensive Care Med 2009;35(10):1738-1748

9 Van den Berghe G, Wilmer A, Hermans G, et al. Intensive insulin therapy in the medical ICU. N Engl J Med 2006;354(5):449-461

10 van den Berghe G, Wouters P, Weekers F, et al. Intensive insulin therapy in critically ill patients. N Engl J Med 2001;345(19): 1359-1367

11 Hoang QN, Pisani MA, Inzucchi S, Hu B, Honiden S. The prevalence of undiagnosed diabetes mellitus and the association of baseline glycemic control on mortality in the intensive care unit: a prospective observational study. J Crit Care 2014;29(6): 1052-1056

12 Krinsley JS, Egi M, Kiss A, et al. Diabetic status and the relation of the three domains of glycemic control to mortality in critically ill patients: an international multicenter cohort study. Crit Care 2013;17(2):R37

13 Siegelaar SE, Hickmann M, Hoekstra JB, Holleman F, DeVries JH. The effect of diabetes on mortality in critically ill patients: a systematic review and meta-analysis. Crit Care 2011;15(5):R205

14 Umpierrez GE, Isaacs SD, Bazargan N, You X, Thaler LM, Kitabchi AE. Hyperglycemia: an independent marker of in-hospital mortality in patients with undiagnosed diabetes. J Clin Endocrinol Metab 2002;87(3):978-982

15 Lang CH, Dobrescu C, Bagby GJ. Tumor necrosis factor impairs insulin action on peripheral glucose disposal and hepatic glucose output. Endocrinology 1992;130(1):43-52

16 Flores EA, Istfan N, Pomposelli JJ, Blackburn GL, Bistrian BR. Effect of interleukin-1 and tumor necrosis factor/cachectin on glucose turnover in the rat. Metabolism 1990;39(7):738-743

17 Khani S, Tayek JA. Cortisol increases gluconeogenesis in humans: its role in the metabolic syndrome. Clin Sci (Lond) 2001;101(6): 739-747

18 Furnary AP, Zerr KJ, Grunkemeier GL, Starr A. Continuous intravenous insulin infusion reduces the incidence of deep sternal wound infection in diabetic patients after cardiac surgical procedures. Ann Thorac Surg 1999;67(2):352-360, discussion 360362

19 Perner A, Nielsen SE, Rask-Madsen J. High glucose impairs superoxide production from isolated blood neutrophils. Intensive Care Med 2003;29(4):642-645

20 Nielson CP, Hindson DA. Inhibition of polymorphonuclear leukocyte respiratory burst by elevated glucose concentrations in vitro. Diabetes 1989;38(8):1031-1035

21 Rassias AJ, Marrin CA, Arruda J, Whalen PK, Beach M, Yeager MP. Insulin infusion improves neutrophil function in diabetic cardiac surgery patients. Anesth Analg 1999;88(5):1011-1016

22 Ling PR, Smith RJ, Bistrian BR. Hyperglycemia enhances the cytokine production and oxidative responses to a low but not high dose of endotoxin in rats. Crit Care Med 2005;33(5): 1084-1089
23 Iwasaki Y, Kambayashi M, Asai M, Yoshida M, Nigawara T, Hashimoto K. High glucose alone, as well as in combination with proinflammatory cytokines, stimulates nuclear factor kappa-B-mediated transcription in hepatocytes in vitro. J Diabetes Complications 2007;21(1):56-62

24 Choi SW, Benzie IF, Ma SW, Strain JJ, Hannigan BM. Acute hyperglycemia and oxidative stress: direct cause and effect? Free Radic Biol Med 2008;44(7):1217-1231

25 Weidig P, McMaster D, Bayraktutan U. High glucose mediates prooxidant and antioxidant enzyme activities in coronary endothelial cells. Diabetes Obes Metab 2004;6(6):432-441

26 West IC. Radicals and oxidative stress in diabetes. Diabet Med 2000;17(3):171-180

27 Carré JE, Orban JC, Re L, et al. Survival in critical illness is associated with early activation of mitochondrial biogenesis. Am J Respir Crit Care Med 2010;182(6):745-751

28 Vanhorebeek I, Gunst J, Derde S, et al. Insufficient activation of autophagy allows cellular damage to accumulate in critically ill patients. J Clin Endocrinol Metab 2011;96(4):E633-E645

29 Rodnick KJ, Piper RC, Slot JW, James DE. Interaction of insulin and exercise on glucose transport in muscle. Diabetes Care 1992; 15(11):1679-1689

30 Devlin JT, Hirshman M, Horton ED, Horton ES. Enhanced peripheral and splanchnic insulin sensitivity in NIDDM men after single bout of exercise. Diabetes 1987;36(4):434-439

31 Kennedy JW, Hirshman MF, Gervino EV, et al. Acute exercise induces GLUT4 translocation in skeletal muscle of normal human subjects and subjects with type 2 diabetes. Diabetes 1999;48(5): 1192-1197

32 Patel BK, Pohlman AS, Hall JB, Kress JP. Impact of early mobilization on glycemic control and ICU-acquired weakness in critically ill patients who are mechanically ventilated. Chest 2014;146(3): 583-589

33 Mashako MN, Bernard C, Cezard JP, Chayvialle JA, Navarro J. Effect of total parenteral nutrition, constant rate enteral nutrition, and discontinuous oral feeding on plasma cholecystokinin immunoreactivity in children. J Pediatr Gastroenterol Nutr 1987;6(6): 948-952

34 Stoll B, Puiman PJ, Cui L, et al. Continuous parenteral and enteral nutrition induces metabolic dysfunction in neonatal pigs. JPEN J Parenter Enteral Nutr 2012;36(5):538-550

35 Hooper MH, Marik PE. Controversies and misconceptions in intensive care unit nutrition. Clin Chest Med 2015;36(3): 409-418

36 MacGregor IL, Gueller R, Watts HD, Meyer JH. The effect of acute hyperglycemia on gastric emptying in man. Gastroenterology 1976;70(2):190-196

37 Oster-Jørgensen E, Pedersen SA, Larsen ML. The influence of induced hyperglycaemia on gastric emptying rate in healthy humans. Scand J Clin Lab Invest 1990;50(8):831-836

38 Samsom M, Akkermans LM, Jebbink RJ, van Isselt H, vanBergeHenegouwen GP, Smout AJ. Gastrointestinal motor mechanisms in hyperglycaemia induced delayed gastric emptying in type I diabetes mellitus. Gut 1997;40(5):641-646

39 Nguyen N, Ching K, Fraser R, Chapman M, Holloway R. The relationship between blood glucose control and intolerance to enteral feeding during critical illness. Intensive Care Med 2007; 33(12):2085-2092

40 Rayner CK, Su YC, Doran SM, Jones KL, Malbert CH, Horowitz M. The stimulation of antral motility by erythromycin is attenuated by hyperglycemia. Am J Gastroenterol 2000;95(9): 2233-2241

41 Van den Berghe G, Wilmer A, Milants I, et al. Intensive insulin therapy in mixed medical/surgical intensive care units: benefit versus harm. Diabetes 2006;55(11):3151-3159

42 Zander R, Boldt J, Engelmann L, Mertzlufft F, Sirtl C, Stuttmann R. The design of the VISEP trial. Critical appraisal [in German]. Anaesthesist 2007;56(1):71-77 
43 Griesdale DE, de Souza RJ, van Dam RM, et al. Intensive insulin therapy and mortality among critically ill patients: a metaanalysis including NICE-SUGAR study data. CMAJ 2009;180(8): 821-827, discussion 799-800

44 Moghissi ES, Korytkowski MT, DiNardo M, et al; American Association of Clinical Endocrinologists; American Diabetes Association. American Association of Clinical Endocrinologists and American Diabetes Association consensus statement on inpatient glycemic control. Endocr Pract 2009;15(4): 353-369

45 Dellinger RP, Levy MM, Rhodes A, et al; Surviving Sepsis Campaign Guidelines Committee including the Pediatric Subgroup. Surviving sepsis campaign: international guidelines for management of severe sepsis and septic shock: 2012. Crit Care Med 2013; 41(2):580-637

46 Falciglia M, Freyberg RW, Almenoff PL, D’Alessio DA, Render ML. Hyperglycemia-related mortality in critically ill patients varies with admission diagnosis. Crit Care Med 2009;37(12): 3001-3009

47 Kosiborod M, Inzucchi SE, Krumholz HM, et al. Glucometrics in patients hospitalized with acute myocardial infarction: defining the optimal outcomes-based measure of risk. Circulation 2008; 117(8):1018-1027

48 Quagliaro L, Piconi L, Assaloni R, Martinelli L, Motz E, Ceriello A. Intermittent high glucose enhances apoptosis related to oxidative stress in human umbilical vein endothelial cells: the role of protein kinase $\mathrm{C}$ and $\mathrm{NAD}(\mathrm{P}) \mathrm{H}$-oxidase activation. Diabetes 2003;52(11):2795-2804

49 Quagliaro L, Piconi L, Assaloni R, et al. Intermittent high glucose enhances ICAM-1, VCAM-1 and E-selectin expression in human umbilical vein endothelial cells in culture: the distinct role of protein kinase $C$ and mitochondrial superoxide production. Atherosclerosis 2005;183(2):259-267

50 Egi M. Blood glucose control in critically ill perioperative patients [in Japanese]. Masui 2011;60(3):285-292

51 Lanspa MJ, Hirshberg EL, Phillips GD, Holmen J, Stoddard G, Orme $\mathrm{J}$. Moderate glucose control is associated with increased mortality compared with tight glucose control in critically ill patients without diabetes. Chest 2013;143(5):1226-1234

52 Kushner M, Nencini P, Reivich M, et al. Relation of hyperglycemia early in ischemic brain infarction to cerebral anatomy, metabolism, and clinical outcome. Ann Neurol 1990;28(2): 129-135

53 Kimura K, Iguchi Y, Inoue T, et al. Hyperglycemia independently increases the risk of early death in acute spontaneous intracerebral hemorrhage. J Neurol Sci 2007;255(1-2):90-94

54 Salim A, Hadjizacharia P, Dubose J, et al. Persistent hyperglycemia in severe traumatic brain injury: an independent predictor of outcome. Am Surg 2009;75(1):25-29

55 Jeremitsky E, Omert LA, Dunham CM, Wilberger J, Rodriguez A. The impact of hyperglycemia on patients with severe brain injury. J Trauma 2005;58(1):47-50

56 Li PA, He QP, Csiszar K, Siesjö BK. Does long-term glucose infusion reduce brain damage after transient cerebral ischemia? Brain Res 2001;912(2):203-205

57 Van den Berghe G, Schoonheydt K, Becx P, Bruyninckx F, Wouters PJ. Insulin therapy protects the central and peripheral nervous system of intensive care patients. Neurology 2005;64(8): 1348-1353

58 Bilotta F, Spinelli A, Giovannini F, Doronzio A, Delfini R, Rosa G. The effect of intensive insulin therapy on infection rate, vasospasm, neurologic outcome, and mortality in neurointensive care unit after intracranial aneurysm clipping in patients with acute subarachnoid hemorrhage: a randomized prospective pilot trial.J Neurosurg Anesthesiol 2007;19(3):156-160

59 Bilotta F, Caramia R, Paoloni FP, Delfini R, Rosa G. Safety and efficacy of intensive insulin therapy in critical neurosurgical patients. Anesthesiology 2009;110(3):611-619
60 Bilotta F, Caramia R, Cernak I, et al. Intensive insulin therapy after severe traumatic brain injury: a randomized clinical trial. Neurocrit Care 2008;9(2):159-166

61 Vespa P, Boonyaputthikul R, McArthur DL, et al. Intensive insulin therapy reduces microdialysis glucose values without altering glucose utilization or improving the lactate/pyruvate ratio after traumatic brain injury. Crit Care Med 2006;34(3):850-856

62 Kosiborod M, Inzucchi SE, Goyal A, et al. Relationship between spontaneous and iatrogenic hypoglycemia and mortality in patients hospitalized with acute myocardial infarction. JAMA 2009; 301(15):1556-1564

63 Krinsley JS, Grover A. Severe hypoglycemia in critically ill patients: risk factors and outcomes. Crit Care Med 2007;35(10): 2262-2267

64 Vriesendorp TM, DeVries JH, van Santen S, et al. Evaluation of short-term consequences of hypoglycemia in an intensive care unit. Crit Care Med 2006;34(11):2714-2718

65 Vriesendorp TM, van Santen S, DeVries JH, et al. Predisposing factors for hypoglycemia in the intensive care unit. Crit Care Med 2006;34(1):96-101

66 Atkin SH, Dasmahapatra A, Jaker MA, Chorost MI, Reddy S. Fingerstick glucose determination in shock. Ann Intern Med 1991;114(12):1020-1024

67 Desachy A, Vuagnat AC, Ghazali AD, et al. Accuracy of bedside glucometry in critically ill patients: influence of clinical characteristics and perfusion index. Mayo Clin Proc 2008;83(4): 400-405

68 Kanji S, Buffie J, Hutton B, et al. Reliability of point-of-care testing for glucose measurement in critically ill adults. Crit Care Med 2005;33(12):2778-2785

69 Egi M, Bellomo R, Stachowski E, French CJ, Hart G. Variability of blood glucose concentration and short-term mortality in critically ill patients. Anesthesiology 2006;105(2):244-252

70 Dossett LA, Cao H, Mowery NT, Dortch MJ, Morris JM Jr, May AK. Blood glucose variability is associated with mortality in the surgical intensive care unit. Am Surg 2008;74(8):679-685, discussion 685

71 Krinsley JS. Glycemic variability: a strong independent predictor of mortality in critically ill patients. Crit Care Med 2008;36(11): 3008-3013

72 Bagshaw SM, Bellomo R, Jacka MJ, Egi M, Hart GK, George C; ANZICS CORE Management Committee. The impact of early hypoglycemia and blood glucose variability on outcome in critical illness. Crit Care 2009;13(3):R91

73 Risso A, Mercuri F, Quagliaro L, Damante G, Ceriello A. Intermittent high glucose enhances apoptosis in human umbilical vein endothelial cells in culture. Am J Physiol Endocrinol Metab 2001; 281(5):E924-E930

74 Monnier L, Mas E, Ginet C, et al. Activation of oxidative stress by acute glucose fluctuations compared with sustained chronic hyperglycemia in patients with type 2 diabetes. JAMA 2006; 295(14):1681-1687

75 Hirsch IB. Glycemic variability: it's not just about A1C anymore! Diabetes Technol Ther 2005;7(5):780-783

76 Hirsch IB, Brownlee M. Should minimal blood glucose variability become the gold standard of glycemic control? J Diabetes Complications 2005;19(3):178-181

77 Wahl HG. How accurately do we measure blood glucose levels in intensive care unit (ICU) patients? Best Pract Res Clin Anaesthesiol 2009;23(4):387-400

78 Blood Glucose Monitoring Test Systems for Prescription Point-ofCare Use; Draft Guidance for Industry and Food and Drug Administration Staff. 2014. Available at: https://www.federalregister.gov/articles/2014/01/07/2014-00023/blood-glucosemonitoring-test-systems-for-prescription-point-of-care-usedraft-guidance-for-industry. Accessed May 25, 2015

79 Center for Clinical Standards and Quality/Survey \& Certification Group. 2015. Available at: http://www.cms.gov/Medicare/ 
Provider-Enrollment-and-Certification/SurveyCertificationGenInfo/Downloads/Survey-and-Cert-Letter-15-11.PDF. Accessed May 25, 2015

80 Klonoff DC, Buckingham B, Christiansen JS, et al; Endocrine Society. Continuous glucose monitoring: an Endocrine Society Clinical Practice Guideline. J Clin Endocrinol Metab 2011;96(10): 2968-2979

81 Joseph JI, Hipszer B, Mraovic B, Chervoneva I, Joseph M, Grunwald Z. Clinical need for continuous glucose monitoring in the hospital. J Diabetes Sci Tech 2009;3(6):1309-1318

82 Tamborlane WV, Beck RW, Bode BW, et al; Juvenile Diabetes Research Foundation Continuous Glucose Monitoring Study Group. Continuous glucose monitoring and intensive treatment of type 1 diabetes. N Engl J Med 2008;359(14): 1464-1476

83 Schierenbeck F, Öwall A, Franco-Cereceda A, Liska J. Evaluation of a continuous blood glucose monitoring system using a central venous catheter with an integrated microdialysis function. Diabetes Technol Ther 2013;15(1):26-31

84 Foubert LA, Lecomte PV, Nobels FR, Gulino AM, De Decker KH. Accuracy of a feasibility version of an intravenous continuous glucose monitor in volunteers with diabetes and hospitalized patients. Diabetes Technol Ther 2014;16(12):858-866

85 Sechterberger MK, van der Voort PH, Strasma PJ, DeVries JH. Accuracy of intra-arterial and subcutaneous continuous glucose monitoring in postoperative cardiac surgery patients in the ICU.J Diabetes Sci Tech 2015;9(3):663-667

86 van Hooijdonk RT, Winters T, Fischer JC, et al. Accuracy and limitations of continuous glucose monitoring using spectroscopy in critically ill patients. Ann Intensive Care 2014;4(1):8

87 De Block C, Manuel-Y-Keenoy B, Van Gaal L, Rogiers P. Intensive insulin therapy in the intensive care unit: assessment by continuous glucose monitoring. Diabetes Care 2006;29(8): 1750-1756

88 Goldberg PA, Siegel MD, Russell RR, et al. Experience with the continuous glucose monitoring system in a medical intensive care unit. Diabetes Technol Ther 2004;6(3):339-347

89 Corstjens AM, Ligtenberg JJ, van der Horst IC, et al. Accuracy and feasibility of point-of-care and continuous blood glucose analysis in critically ill ICU patients. Crit Care 2006;10(5):R135

90 Brunner R, Kitzberger R, Miehsler W, Herkner H, Madl C, Holzinger $U$. Accuracy and reliability of a subcutaneous continuous glucose-monitoring system in critically ill patients. Crit Care Med 2011;39(4):659-664

91 Kopecký P, Mráz M, Bláha J, et al. The use of continuous glucose monitoring combined with computer-based eMPC algorithm for tight glucose control in cardiosurgical ICU. Biomed Res Int 2013; 2013:186439

92 Kosiborod M, Gottlieb RK, Sekella JA, et al. Performance of the Medtronic Sentrino continuous glucose management (CGM) system in the cardiac intensive care unit. BMJ Open Diabetes Res Care 2014;2(1):e000037

93 Schuster KM, Barre K, Inzucchi SE, Udelsman R, Davis KA. Continuous glucose monitoring in the surgical intensive care unit: concordance with capillary glucose. J Trauma Acute Care Surg 2014;76(3):798-803
94 Clarke WL, Cox D, Gonder-Frederick LA, Carter W, Pohl SL. Evaluating clinical accuracy of systems for self-monitoring of blood glucose. Diabetes Care 1987;10(5):622-628

95 Rabiee A, Andreasik V, Abu-Hamdah R, et al. Numerical and clinical accuracy of a continuous glucose monitoring system during intravenous insulin therapy in the surgical and burn intensive care units. J Diabetes Sci Tech 2009;3(4):951-959

96 Boom DT, Sechterberger MK, Rijkenberg S, et al. Insulin treatment guided by subcutaneous continuous glucose monitoring compared to frequent point-of-care measurement in critically ill patients: a randomized controlled trial. Crit Care 2014;18(4):453

97 Holzinger U, Warszawska J, Kitzberger R, et al. Real-time continuous glucose monitoring in critically ill patients: a prospective randomized trial. Diabetes Care 2010;33(3):467-472

98 Breton M, Farret A, Bruttomesso D, et al; International Artificial Pancreas Study Group. Fully integrated artificial pancreas in type 1 diabetes: modular closed-loop glucose control maintains near normoglycemia. Diabetes 2012;61(9):2230-2237

99 Krenitsky J. Glucose control in the intensive care unit: a nutrition support perspective. Nutr Clin Pract 2011;26(1):31-43

100 Rice TW, Wheeler AP, Thompson BT, et al; National Heart, Lung, and Blood Institute Acute Respiratory Distress Syndrome (ARDS) Clinical Trials Network. Initial trophic vs full enteral feeding in patients with acute lung injury: the EDEN randomized trial. JAMA 2012;307(8):795-803

101 Hsu CW, Sun SF, Lin SL, Huang HH, Wong KF. Moderate glucose control results in less negative nitrogen balances in medical intensive care unit patients: a randomized, controlled study. Crit Care 2012;16(2):R56

102 Leite HP, de Lima LF, de Oliveira Iglesias SB, Pacheco JC, de Carvalho WB. Malnutrition may worsen the prognosis of critically ill children with hyperglycemia and hypoglycemia. JPEN J Parenter Enteral Nutr 2013;37(3):335-341

103 Kauffmann RM, Hayes RM, VanLaeken AH, et al. Hypocaloric enteral nutrition protects against hypoglycemia associated with intensive insulin therapy better than intravenous dextrose. Am Surg 2014;80(11):1106-1111

104 de Azevedo JR, de Araujo LO, da Silva WS, de Azevedo RP. A carbohydrate-restrictive strategy is safer and as efficient as intensive insulin therapy in critically ill patients. J Crit Care 2010;25(1):84-89

105 Mesejo A, Acosta JA, Ortega C, et al. Comparison of a high-protein disease-specific enteral formula with a high-protein enteral formula in hyperglycemic critically ill patients. Clin Nutr 2003; 22(3):295-305

106 Déchelotte P, Hasselmann M, Cynober L, et al. L-alanyl-L-glutamine dipeptide-supplemented total parenteral nutrition reduces infectious complications and glucose intolerance in critically ill patients: the French controlled, randomized, double-blind, multicenter study. Crit Care Med 2006;34(3):598-604

107 Bakalar B, Duska F, Pachl J, et al. Parenterally administered dipeptide alanyl-glutamine prevents worsening of insulin sensitivity in multiple-trauma patients. Crit Care Med 2006;34(2):381-386

108 Heyland D, Muscedere J, Wischmeyer PE, et al; Canadian Critical Care Trials Group. A randomized trial of glutamine and antioxidants in critically ill patients. N Engl J Med 2013;368(16):1489-1497 